\title{
Satisfação de familiares de pacientes psiquiátricos com os serviços de saúde mental e seus fatores associados
}

\author{
Family satisfaction in mental health services and associated factors \\ Marina Bandeira', Mônia Aparecida da Silva², Cleucimara Aparecida Camilo33, Cynthia Mara Felício
}

\section{RESUMO}

Objetivo: Avaliar a satisfação de familiares cuidadores de pacientes psiquiátricos com os serviços de saúde mental e seus fatores associados. Método: Realizou-se uma pesquisa avaliativa de serviços, do tipo correlacional e de corte transversal, com uma amostra de 85 familiares cuidadores de pacientes psiquiátricos, atendidos em três serviços de saúde mental públicos, situados em três cidades do interior de Minas Gerais. Utilizaram-se a Escala de Avaliação da Satisfação dos Familiares com os Serviços de Saúde Mental (SATIS-BR) e um questionário de variáveis sociodemográficas e clínicas. Resultados: A maioria dos familiares estava satisfeita ou muito satisfeita em relação aos aspectos avaliados dos serviços. A satisfação estava significativa e positivamente associada à idade dos pacientes e ao número de meses sem internação psiquiátrica. Não foi detectada influência do tipo de serviço no grau de satisfação. Conclusão: As variáveis dos pacientes foram os principais fatores associados à satisfação dos familiares. A satisfação dos familiares foi elevada, tendo-se inferido a influência parcial do contraste com outros serviços de saúde. Foi apontada a necessidade de maior diferenciação entre os serviços. Estudos futuros, com amostras maiores e aleatórias, poderão reavaliar esses resultados.

\section{ABSTRACT}

Objective: To evaluate family caregivers' satisfaction with mental health services and associated factors. Method: A cross-sectional correlational study of service evaluation was carried out with a sample of 85 family caregivers of psychiatric patients attending three public mental health services located in three towns in the state of Minas Gerais. Subjects were interviewed for the application of the Satisfaction with Mental Health Services Scale (SATIS-BR) and a sociodemographic and clinic variables questionnaire. Results: Results showed that most caregivers were satisfied or very satisfied with the services aspects evaluated. Family satisfaction was significantly and positively associated with patients' age and time spent without hospitalization. The statistical analysis did not detect any effect of type of service on family satisfaction. Conclusion: Patients' variables were the most important associated factors related to the family satisfaction. Family satisfaction was high, maybe due partly to a comparison with other health services. Data suggest the need for more differentiation among services. Future studies with bigger and randomized samples will be able to reevaluate theses results.

1 Universidade Federal de São João del Rei (UFSJ); Université de Montreal, McGill University.

2 UFSJ, Programa de Mestrado em Psicologia.

3 UFSJ, Programa de Mestrado em Psicologia, Ambulatório de Saúde Mental de Nepomuceno.

Recebido em

17/10/2011

Aprovado em 4 UFSJ.

Endereço para correspondência: Marina Bandeira

Departamento de Psicologia - Laboratório de Pesquisa em Saúde Mental (LAPSAM) - UFSJPraça Dom Helvécio, 74, Dom Bosco São 36301-160 - João del Rei, MG

E-mail:bandeira@ufsj.edu.br 


\section{INTRODUÇÃO}

A inclusão da perspectiva dos usuários no monitoramento e avaliação dos serviços de saúde mental tem sido altamente recomendada, visando assegurar que os cuidados prestados por esses serviços sejam adequados às necessidades dos pacientes e de seus familiares ${ }^{1,2}$. Segundo Donabedian ${ }^{3}$, para que os serviços de saúde sejam de qualidade, os resultados devem ser congruentes com as perspectivas dos usuários. Além disso, as avaliações subjetivas dos usuários apresentam a vantagem de aferir o impacto real das mudanças produzidas pelo tratamento na vida dos pacientes ${ }^{4}$. Incluir as perspectivas dos usuários na avaliação dos serviços fornece uma informação valiosa, pois muitas vezes não há uma correspondência direta entre o que os usuários percebem como mudança desejável e os resultados objetivos mensurados por terceiros. Pequenas mudanças produzidas pelo tratamento podem fazer uma grande diferença para os usuários, enquanto mudanças significativas em medidas clínicas objetivas podem ser consideradas irrelevantes para eles ${ }^{5}$.

O envolvimento dos familiares, em colaboração com os profissionais dos serviços, apresenta vantagens para o sucesso do tratamento, estando associado à melhora no funcionamento social dos pacientes, à diminuição da duração das internações, à redução de conflitos familiares e à diminuição da sobrecarga dos familiares cuidadores dos pacientes ${ }^{6,7}$. Perreault et al. ${ }^{7}$ apontaram para a necessidade de aumentar a participação dos familiares no tratamento e na avaliação dos serviços, tendo em vista que essa colaboração ainda deixa a desejar e que os familiares se ressentem com isso. As queixas dos familiares estão relacionadas com a falta de informação sobre os transtornos psiquiátricos e o tratamento dos pacientes e com a falta de orientações sobre como agir com os pacientes, no dia a dia e nos momentos de crise ${ }^{7,8}$.

Utilizar as avaliações dos familiares sobre os resultados do tratamento pode ser particularmente útil, porque eles são os principais provedores de cuidados aos pacientes, nas atividades cotidianas, na supervisão aos comportamentos problemáticos e na tomada dos medicamentos, que afetam os próprios resultados do tratamento ${ }^{9,10}$. Eles podem, portanto, detectar mudanças em virtude do tratamento, em diversos aspectos da vida dos pacientes, e essas informações ajudam os profissionais a identificarem os aspectos do tratamento que já atendem às necessidades dos pacientes e aqueles que ainda necessitam ser melhorados para atingir esses objetivos. Além disso, incluir os familiares na avaliação do tratamento pode ter um efeito positivo em seu bem-estar e sentimento de empoderamento, podendo diminuir seu grau de sobrecarga com o papel de cuidador, segundo Perreault et al. ${ }^{7}$ e Tessler e Gamache ${ }^{9}$.

Uma das medidas mais utilizadas para a avaliação da qualidade dos serviços de saúde mental consiste na satisfação dos usuários ${ }^{11}$. A satisfação dos usuários tem sido associada com melhor adesão ao tratamento e menor taxa de abandono ${ }^{2,11,12}$, além de ser um fator preditivo para menor taxa de hospitalizações futuras dos pacientes ${ }^{13}$. Hanson ${ }^{2}$ destaca que as avaliações subjetivas de satisfação dos usuários com os serviços são importantes, porque estão mais associadas com a utilização de serviços do que as medidas objetivas tomadas por terceiros. O conceito de satisfação tem sido formulado por diferentes modelos explicativos, sendo o mais utilizado o modelo da expectativa, segundo o qual o usuário avalia o serviço a partir de uma comparação que ele faz entre as expectativas que possuía previamente e os resultados reais de sua experiência com o serviço. Três modelos de expectativa foram formulados, dentre os quais o modelo do contraste, que postula que a satisfação ocorre quando o usuário observa que sua experiência atual com um determinado produto ou serviço está acima das suas experiências prévias, em situações semelhantes ${ }^{14}$.

Há um consenso de que a satisfação é um construto multidimensional, devendo, portanto, ser avaliado por medidas multifatoriais. Medidas multifatoriais, que são compostas por subescalas, são importantes para detectar aspectos específicos dos serviços que determinam a insatisfação ou a satisfação, o que não pode ser avaliado com medidas globais. Avaliações de serviços devem também ser feitas com medidas padronizadas, com suas propriedades psicométricas avaliadas, visando favorecer a validade dos dados coletados e garantir a possibilidade de comparação entre os estudos ${ }^{11,14}$.

A maioria das pesquisas de avaliação de serviços de saúde mental tem focalizado a satisfação dos pacientes. Pouca ênfase tem sido dada à avaliação da satisfação dos familiares com esses serviços, apesar das recomendações feitas sobre a necessidade da inclusão dos familiares no monitoramento e avaliação dos serviços ${ }^{18,15}$.

Alguns estudos internacionais levantaram os fatores associados à satisfação dos familiares com os serviços e com o papel de cuidador. Stengard et al. ${ }^{8}$ entrevistaram, na Finlândia, uma amostra de 545 familiares de pacientes com esquizofrenia, deinstitucionalizados, após três anos de sua alta do hospital, usando uma medida de um único escore global de três pontos para avaliar a satisfação com os serviços. O estudo constatou que um terço da amostra estava insatisfeita com os serviços de saúde mental. A insatisfação era maior quando os pacientes recebiam menos tratamento ou frequentavam menos o serviço, apresentavam sintomatologia psicótica mais severa e menor nível de autonomia. Os autores concluíram que a satisfação dos familiares estava associada a dois fatores principais: ter uma participação mais ativa como colaboradores dos profissionais do serviço e ter serviços que ajudem o paciente a atingir o máximo de autonomia possível. 
Perreault et al. ${ }^{7}$ analisaram, por meio de regressão múltipla, a influência relativa de diversas variáveis na determinação da satisfação com os serviços, em uma amostra de 145 familiares canadenses, tendo encontrado que o fator preditivo mais importante era o grau de colaboração entre familiares e profissionais. Além disso, a satisfação dos familiares era maior quando eles tinham um maior número de necessidades de tratamento preenchidas, menor grau de sobrecarga objetiva e subjetiva e quando os pacientes apresentavam menor número de comportamentos problemáticos. Observou-se que os familiares estavam mais satisfeitos com o relacionamento com os profissionais do que com as características da estrutura e organização dos serviços, o que confirma, segundo os autores, a tendência dos resultados dos estudos que avaliaram a satisfação dos próprios pacientes com os serviços.

No contexto brasileiro, foram encontrados apenas quatro estudos, publicados em periódicos científicos indexados, que avaliaram a satisfação dos usuários com os serviços de saúde mental, utilizando escalas de medidas validadas para aferir a satisfação. Apenas um desses estudos focalizou a satisfação dos familiares, porém com o objetivo de fazer a validação de uma escala de medida ${ }^{16}$, e os três outros estudos avaliaram a satisfação dos pacientes ${ }^{17-19}$. Tendo em vista a carência de estudos com os familiares e a importância dessa temática, a presente pesquisa teve como objetivo avaliar a satisfação de familiares de pacientes psiquiátricos em relação aos serviços de saúde mental e seus fatores associados.

\section{MÉTODO}

\section{Delineamento do estudo}

Trata-se de um estudo correlacional visando avaliar os fatores associados à satisfação dos familiares com os serviços de saúde mental. A pesquisa é de corte transversal, realizada a partir de dados coletados em uma única entrevista estruturada, com aplicação de uma escala de medida multifatorial da satisfação dos familiares.

Consiste, também, em uma pesquisa avaliativa, do tipo somativa, definida como aquela que visa avaliar os efeitos de serviços ou programas de tratamento, segundo a classificação de Selltiz et al..$^{20}$ e de Contandriopoulos et al. ${ }^{21}$. No caso deste estudo, avaliou-se a satisfação dos familiares com os serviços oferecidos aos pacientes, em relação às dimensões dos resultados do tratamento, dos cuidados prestados e competência da equipe, da privacidade e confidencialidade dos serviços.

\section{Participantes}

Participaram deste estudo 85 familiares de pacientes psiquiátricos que estavam sendo atendidos em três serviços de saúde mental de diferentes municípios do interior de Mi- nas Gerais. Houve a participação de apenas um familiar por paciente. Foi utilizada uma amostra não probabilística de familiares, selecionados por meio de indicações dos profissionais dos serviços, que preenchiam os seguintes critérios de inclusão: ter idade igual ou superior a 18 anos, ser indicado como principal cuidador do paciente e conviver diariamente com ele. Foram excluídos os familiares que apresentavam transtornos psiquiátricos e que não tinham condições de compreender as questões dos instrumentos utilizados. Os serviços participantes desta pesquisa incluíram: dois Centros de Atenção Psicossocial (um CAPS tipo I e um CAPS tipo II) e um Ambulatório de Saúde Mental. Apesar de a amostra ser constituída, em geral, por familiares de pacientes de CAPS, a maioria dos pacientes estava sendo atendida em regime não intensivo, que inclui apenas consultas mensais, para acompanhamento da medicação aos pacientes com quadro clínico estável.

\section{Instrumentos de medida}

Escala de avaliação da satisfação dos familiares: A satisfação dos familiares foi avaliada por meio da versão abreviada da Escala de Avaliação da Satisfação dos Familiares com os Serviços de Saúde Mental (SATIS-BR). Essa escala foi elaborada pela Divisão de Saúde Mental da Organização Mundial de Saúde ${ }^{22}$ e validada, para o Brasil, por Bandeira et al. ${ }^{16}$.

Essa escala possui oito itens agrupados em três subescalas: 1. Resultados do tratamento; 2. Acolhida e competência da equipe; e 3. Privacidade e confidencialidade do serviço. As alternativas de resposta aos itens estão dispostas em uma escala do tipo Likert, com 5 pontos ( 1 = muito insatisfeitos e 5 = muito satisfeitos). A escala SATIS-BR apresentou bons indicadores de consistência interna, avaliada pela análise da alfa de Cronbach, tendo obtido um valor de alfa de 0,79 para o escore global e valores entre 0,76 e 89 para suas subescalas. Esses valores situam-se dentro da faixa considerada ideal $(0,75$ a $0,85)$ por Vallerand ${ }^{23}$, indicando que as questões da escala são homogêneas, sem serem repetitivas. A validade de construto da escala foi avaliada pela análise de sua estrutura dimensional, pelo método dos Componentes Principais, com rotação Varimax, tendo sido obtidos três fatores, com eigenvalues acima de 1.0, que explicaram 77\% da variância dos dados ${ }^{16}$.

Questionário sociodemográfico e clínico: Os dados sociodemográficos dos familiares e dos pacientes e os dados clínicos dos pacientes foram coletados por meio de um questionário, previamente testado em um estudo piloto, visando avaliar a compreensão das questões pela população-alvo. O questionário incluía questões sobre as seguintes variáveis: idade, gênero, data de nascimento, estado civil, escolaridade, renda, tipo de trabalho e parentesco com o paciente. As variáveis clínicas dos pacientes avaliadas foram: tipo de diagnóstico, presença de comorbidade psiquiátrica, duração do transtorno, duração do tratamento psiquiátrico, número de internações, tipo de medicação utilizada, existência de 
doenças físicas e número de crises no último ano. Dados clínicos referentes ao diagnóstico, comorbidades, tempo de tratamento e tipo de medicação foram identificados nos prontuários dos pacientes.

\section{Coleta de dados}

Os dados foram coletados por meio de entrevistas estruturadas, com duração aproximada de 30 minutos, para aplicação dos instrumentos de medida, por estagiários de Psicologia previamente treinados. As entrevistas foram realizadas nos serviços de saúde mental, quando os familiares se apresentavam para acompanhar os pacientes, ou em suas residências, após agendamento prévio e conforme sua disponibilidade. A compreensão dos familiares acerca das questões da escala de satisfação foi verificada por meio da Técnica de Sondagem (Probe Technique) ${ }^{24}$, que consiste em solicitar aos sujeitos que justifiquem suas respostas a cada um dos itens da escala.

O presente estudo foi aprovado pela Comissão de Ética em Pesquisa Envolvendo Seres Humanos (CEPES) da UFSJ, processo no 016-a/2010/CEPES, e pela direção das instituições participantes. Os entrevistados foram informados sobre os objetivos do estudo e sobre o sigilo e anonimato dos resultados, tendo assinado um Termo de Consentimento Livre e Esclarecido concordando em participar da pesquisa. Os participantes receberam uma cópia desse documento, com um número de telefone para contato com os pesquisadores.

\section{Análise dos dados}

Os dados foram computados e analisados por meio do programa SPSS, versão 13.0. As análises estatísticas foram propostas com base nas obras de Bisqueira et al. ${ }^{25}$ e Dancey e Reidy ${ }^{26}$. Foram utilizados testes estatísticos não paramétricos, uma vez que os dados não apresentavam distribuição normal, conforme avaliado pelo teste Kolmogorov-Smirnov (KS: 1,$53 ; p=0,02$ ).

Para a descrição das características sociodemográficas e clínicas da amostra e avaliação dos escores de satisfação global, por subescalas e por itens, foi utilizada a análise estatística descritiva, com cálculo de médias, desvios-padrão (DP) e porcentagens. Foram também calculadas as porcentagens de familiares que se declararam insatisfeitos, mais ou menos insatisfeitos ou satisfeitos com os serviços. Os escores médios das três subescalas da SATIS-BR foram comparados, utilizando-se os testes não paramétricos de Kendall's W para verificar se havia diferença significativa entre eles. Além disso, essas médias foram comparadas duas a duas, pelo teste de Wilcoxon para duas amostras relacionadas, visando identificar quais pares de subescalas apresentavam diferenças significativas. Para identificar os itens específicos da escala que apresentavam escores médios mais elevados de satisfação, foi feita uma análise da média de postos, pelo teste não paramétrico de Friedman. Foi utilizado o nível de significância de $p<0,05$.

Foi realizada também uma análise estatística univariada, para identificar as variáveis clínicas dos pacientes e as variáveis sociodemográficas dos pacientes e familiares, associadas à maior satisfação dos familiares com os serviços de saúde mental. Para as variáveis categóricas, foram utilizados o teste Man-Whitney para comparar dois grupos e o teste de Kruskal-Wallis para comparar mais de dois grupos. Para as variáveis contínuas, foi utilizada a correlação de Spearman. Não foi feita uma análise multivariada de regressão múltipla, tendo em vista que os dados dos escores de satisfação não apresentaram distribuição normal.

\section{RESULTADOS}

\section{Descrição das amostras}

As tabelas 1 e 2 mostram os principais dados descritivos da amostra dos familiares avaliados e as características dos pacientes dos quais eles cuidavam. A maioria dos familiares era do gênero feminino (80,00\%), vivendo com companheiro em união estável (60,00\%), com escolaridade de ensino fundamental (71,80\%). A idade média era de 54,61 anos, sendo a idade mínima de 19 e a máxima de 78 anos. A maioria dos familiares não possuía trabalho formal (79,60\%), mas tinha renda própria (62,40\%), de um a dois salários-mínimos (79,30\%), originada principalmente de aposentadoria (54,80\%). Com relação ao grau de parentesco, as mães e pais eram os principais cuidadores dos pacientes (43,60\%).

Os pacientes que recebiam cuidados dos familiares estudados eram, em sua maioria, do gênero feminino $(57,60 \%)$ e com escolaridade de ensino fundamental (78,70\%). A idade média era de 43,53 anos, sendo a mínima de 20 e a máxima de 73 anos. Grande parte possuía renda própria (62,40\%), cujo valor era, na maioria dos casos $(79,30 \%)$, de um a dois salários-mínimos e as principais fontes de renda eram a aposentadoria $(49,00 \%)$ e o auxílio do governo (20,80\%). A maioria dos pacientes possuía diagnóstico da categoria "Esquizofrenia, Transtornos esquizotípicos e Transtornos delirantes" (60,00\%), não apresentava comorbidade psiquiátrica $(81,20 \%)$ e não possuía doenças físicas $(55,30 \%)$. Metade dos pacientes $(50,60 \%)$ não havia apresentado crises no último ano e $36,50 \%$ nunca tinham sido internados em hospitais psiquiátricos. A idade média de início do transtorno psiquiátrico era de 27,10 anos; a duração média do transtorno era de 16,89 anos; e a duração média do tratamento psiquiátrico era de 13,02 anos. Os pacientes tomavam, em média, 2,99 tipos de medicamentos por dia. A maioria tomava a medicação de forma independente, sem a ajuda do familiar $(65,90 \%)$. A maioria estava em regime de tratamento psiquiátrico não intensivo (56,50\%). 
Tabela 1. Principais características sociodemográficas dos familiares e dos pacientes

\begin{tabular}{|c|c|c|c|}
\hline Variáveis & Categorias & $\begin{array}{c}\text { Familiares } \\
\text { Frequência (\%) }\end{array}$ & $\begin{array}{c}\text { Pacientes } \\
\text { Frequência (\%) }\end{array}$ \\
\hline Gênero & $\begin{array}{l}\text { Feminino } \\
\text { Masculino }\end{array}$ & $\begin{array}{l}68(80,00 \%) \\
17(20,00 \%)\end{array}$ & $\begin{array}{l}49(57,60 \%) \\
36(42,40 \%)\end{array}$ \\
\hline Estado civil & $\begin{array}{l}\text { Solteiro } \\
\text { Casado/Vivendo como casado } \\
\text { Separado/divorciado/viúvo }\end{array}$ & $\begin{array}{l}11(12,90 \%) \\
51(60,00 \%) \\
23(27,10 \%)\end{array}$ & \\
\hline Nível de escolaridade & $\begin{array}{l}\text { Analfabeto } \\
\text { Ensino fundamental completo ou incompleto } \\
\text { Ensino médio completo ou incompleto } \\
\text { Pós-graduação }\end{array}$ & $\begin{array}{l}12(14,10 \%) \\
61(71,80 \%) \\
12(14,10 \%) \\
-\end{array}$ & $\begin{array}{c}5(5,90 \%) \\
67(78,70 \%) \\
10(11,80 \%) \\
3(3,60 \%)\end{array}$ \\
\hline Trabalho formal & $\begin{array}{l}\text { Sim } \\
\text { Não }\end{array}$ & $\begin{array}{l}11(20,40 \%) \\
74(79,60 \%)\end{array}$ & $\begin{array}{c}7(13,20 \%) \\
78(86,80 \%)\end{array}$ \\
\hline Possui renda própria & $\begin{array}{l}\text { Sim } \\
\text { Não }\end{array}$ & $\begin{array}{l}53(62,40 \%) \\
32(37,60 \%)\end{array}$ & $\begin{array}{l}53(62,40 \%) \\
32(37,60 \%)\end{array}$ \\
\hline Renda (em salários-mínimos) & $\begin{array}{l}<1 \\
1 \text { a } 2 \\
2,1 \text { a } 3 \\
3,1 \text { a } 4\end{array}$ & $\begin{array}{c}5(9,40 \%) \\
42(79,30 \%) \\
5(9,40 \%) \\
1(1,90 \%)\end{array}$ & $\begin{array}{c}5(9,40 \%) \\
42(79,30 \%) \\
5(9,40 \%) \\
1(1,90 \%)\end{array}$ \\
\hline Origem da renda & $\begin{array}{l}\text { Emprego ou trabalho regular } \\
\text { Aposentadoria } \\
\text { Pensão } \\
\text { Auxílio do governo/Encostado } \\
\text { Outra }\end{array}$ & $\begin{array}{c}11(20,70 \%) \\
29(54,80 \%) \\
11(20,70 \%) \\
1(1,90 \%) \\
1(1,90 \%)\end{array}$ & $\begin{array}{c}7(13,20 \%) \\
26(49,00 \%) \\
8(15,10 \%) \\
11(20,80 \%) \\
1(1,90 \%)\end{array}$ \\
\hline Parentesco entre familiar e paciente & $\begin{array}{l}\text { Mãe ou pai } \\
\text { Irmão(a) } \\
\text { Cônjuge } \\
\text { Filho(a) } \\
\text { Outro }\end{array}$ & $\begin{array}{c}37(43,60 \%) \\
17(20,00 \%) \\
15(17,60 \%) \\
8(9,40 \%) \\
8(9,40 \%)\end{array}$ & \\
\hline
\end{tabular}

Tabela 2. Principais características clínicas dos pacientes

\begin{tabular}{|c|c|c|c|}
\hline Variáveis & Categorias & Frequência (\%) & Média \pm desvio-padrão \\
\hline Categoria diagnóstica & $\begin{array}{l}\text { - Esquizofrenia, transtornos esquizotípicos } \\
\text { e delirantes } \\
\text { - Transtornos do humor e afetivos } \\
\text { - Transtornos neuróticos relacionados ao } \\
\text { estresse e somatoforme } \\
\text { - Transtornos fóbicos e ansiosos } \\
\text { - Diagnóstico não especificado }\end{array}$ & $\begin{array}{c}51(60,00 \%) \\
23(27,00 \%) \\
8(9,40 \%) \\
1(1,20 \%) \\
2(2,40 \%)\end{array}$ & \\
\hline Comorbidade psiquiátrica & $\begin{array}{l}\text { Sim } \\
\text { Não } \\
\text { Não especificado }\end{array}$ & $\begin{array}{c}15(17,60 \%) \\
69(81,20 \%) \\
1(1,20)\end{array}$ & \\
\hline Regime de tratamento & $\begin{array}{l}\text { CAPS dia } \\
\text { Ambulatorial }\end{array}$ & $\begin{array}{l}37(43,50 \%) \\
48(56,50 \%)\end{array}$ & \\
\hline Idade de início do transtorno psiquiátrico & & & $27,10 \pm 11,88$ anos \\
\hline Duração do transtorno & & & $16,89 \pm 10,32$ anos \\
\hline Duração do tratamento psiquiátrico & & & $13,02 \pm 9,33$ anos \\
\hline Crises no último ano & $\begin{array}{l}\text { Não teve crises } \\
\text { Teve } 1 \text { crise } \\
\text { Teve de } 2 \text { a } 5 \text { crises } \\
\text { Teve de } 6 \text { a } 10 \text { crises }\end{array}$ & $\begin{array}{c}43(50,60 \%) \\
21(24,70 \%) \\
17(20,00 \%) \\
4(4,70 \%)\end{array}$ & $2,74 \pm 2,38$ crises \\
\hline Internações & $\begin{array}{l}\text { Nunca foi internado } \\
1 \text { a } 5 \text { internações } \\
6 \text { a } 10 \text { internações } \\
\text { Mais de } 10 \text { internações } \\
\text { Não souberam informar }\end{array}$ & $\begin{array}{c}31(36,50 \%) \\
35(41,20 \%) \\
11(12,90 \%) \\
7(8,20 \%) \\
1(1,20 \%)\end{array}$ & $7,42 \pm 18,00$ internações \\
\hline Número de tipos de medicamentos psiquiátricos & & & $2,99 \pm 1,21$ tipos \\
\hline Toma o medicamento sozinho & $\begin{array}{l}\text { Sim } \\
\text { Não }\end{array}$ & $\begin{array}{l}56(65,90 \%) \\
29(34,10 \%)\end{array}$ & \\
\hline Doenças físicas & $\begin{array}{l}\text { Sim } \\
\text { Não }\end{array}$ & $\begin{array}{l}38(44,70 \%) \\
47(55,30 \%)\end{array}$ & \\
\hline
\end{tabular}




\section{Satisfação dos familiares com os serviços}

Análise por subescalas: A tabela 3 apresenta os escores médios de satisfação dos familiares, para a escala global e para as três subescalas da SATIS-BR e os valores de $p$ para a comparação entre elas, duas a duas, por meio do teste não paramétrico de Wilcoxon para amostras relacionadas. $O$ escore médio de satisfação global foi de 4,41 (DP =0,56), em uma faixa de variação entre 1 e 5, indicando que, em geral, os familiares dos pacientes estavam entre satisfeitos e muito satisfeitos com os serviços.

Os escores médios de satisfação foram elevados também para as subescalas, sendo 4,55 para a subescala 1, "Resultados do tratamento", 4,29 para a subescala 2, "Acolhida e competência da equipe" e 4,40 para a subescala 3, "Privacidade e confidencialidade do serviço". Uma comparação dessas médias, pelo teste não paramétrico de Kendall's W, indicou uma diferença significativa entre elas $\left(X^{2}=26,85\right.$; $p<0,001)$. A comparação das médias das subescalas duas a duas, pelo teste de Wilcoxon, confirmou que os familiares estavam significativamente mais satisfeitos em relação aos "Resultados do tratamento", comparativamente à "Acolhida e competência da equipe" $(p<0,001)$ e à "Privacidade e confidencialidade do serviço" $(p<0,01)$. Não houve diferença estatisticamente significativa da satisfação dos familiares entre as subscalas 2 e $3(p=0,09)$.

Tabela 3. Médias de satisfação dos familiares por subescalas e escala global e comparação das médias das subescalas, duas a duas, pelo teste não paramétrico de Wilcoxon

\begin{tabular}{lllll}
\hline \multirow{2}{*}{ Subescalas } & Média (DP) & \multicolumn{3}{c}{ Wilcoxon } \\
\cline { 3 - 5 } & & $\mathrm{p}(1-2)$ & $\mathrm{p}(1-3)$ & $\mathrm{p}(2-3)$ \\
\hline 1. Resultados do tratamento & $4,55(0,64)$ & & & \\
2. Acolhida e competência da equipe & $4,29(0,64)$ & $0,00^{* *}$ & & 0,09 \\
3. Privacidade e confidencialidade do serviço & $4,40(0,59)$ & & $0,00^{*}$ & \\
Escala global & $4,41(0,56)$ & & \\
\hline
\end{tabular}

** $p<0,001 ; *<0,01 ; p(1-2)$ : comparação das subescalas 1 e 2; $p(1-3)$ : comparação das subescalas 1 e 3 ; $p(2-3)$ : comparação das subescalas 2 e 3; DP: desvio-padrão.
Análise por itens: A tabela 4 apresenta os resultados descritivos dos escores de satisfação dos familiares para cada item da escala SATIS-BR, em termos das porcentagens de familiares insatisfeitos, mais ou menos satisfeitos e satisfeitos com os serviços. Os resultados mostram que a maioria dos familiares estava satisfeita ou muito satisfeita (respostas 4 e 5) em relação aos diversos aspectos avaliados (81,20\% a 96,40\%). Os itens que apresentaram maiores porcentagens de satisfação dos familiares foram: as medidas tomadas para assegurar a privacidade do tratamento (item 5), a confidencialidade das informações (item 6), a competência do profissional (item 7) e a capacidade dos serviços em ajudar o paciente a lidar mais eficientemente com os seus problemas (item 3).

No que se refere às porcentagens mais elevadas de insatisfação, destacaram-se os itens: compreensão do paciente pelo profissional que o admitiu (item 1), compreensão pela equipe do tipo de ajuda que o paciente necessitava (item 2) e obtenção do tipo de ajuda de que o paciente necessitava (item 4). Nenhum familiar estava insatisfeito em relação à competência do terapeuta principal.

A tabela 4 apresenta também os dados referentes à comparação dos escores médios de satisfação aos itens específicos da escala, por meio de uma análise da média de postos de Friedman. Os resultados mostraram uma diferença significativa entre os escores de satisfação dos familiares nos oito itens da escala ( $\left.X^{2}=68,16 ; p<0,001\right)$. As médias mais elevadas de satisfação se concentraram principalmente nos itens que avaliaram: os benefícios que o paciente recebeu com o tratamento (item 8), se os serviços recebidos ajudaram o paciente a lidar mais eficientemente com seus problemas (item 3), a competência do terapeuta principal (item 7) e se o paciente obteve o tipo de ajuda de que necessitava (item 4).

\section{Fatores associados à satisfação dos familiares}

Para a análise dos fatores associados à satisfação dos familiares, algumas categorias de variáveis foram agrupadas e outras não foram investigadas, por causa da ocorrência de

Tabela 4. Porcentagens de resposta dos familiares para cada item da escala SATIS-BR e médias de postos de Friedman

\begin{tabular}{|c|c|c|c|c|}
\hline \multirow{3}{*}{ Itens } & Insatisfeitos & $\begin{array}{l}\text { Mais ou menos } \\
\text { satisfeitos }\end{array}$ & Satisfeitos & \multirow{3}{*}{ Média de postos } \\
\hline & Respostas & Respostas & Respostas & \\
\hline & $1 e 2$ & 3 & 4 e 5 & \\
\hline 1. Compreensão do paciente pelo profissional que 0 admitiu & $5(5,90 \%)$ & $11(12,90 \%)$ & $69(81,20 \%)$ & 3,69 \\
\hline 2. Equipe compreendeu o tipo de ajuda de que o paciente necessitava & $1(1,20 \%)$ & $14(16,50 \%)$ & $70(82,30 \%)$ & 3,86 \\
\hline 3. Serviços recebidos ajudaram o paciente a lidar melhor com os seus problemas & $1(1,20 \%)$ & $4(4,70 \%)$ & $80(94,10 \%)$ & 5,27 \\
\hline 4. Obtenção do tipo de ajuda de que o paciente necessitava & $3(3,50 \%)$ & $9(10,60 \%)$ & $73(85,90 \%)$ & 4,46 \\
\hline 5. Medidas tomadas para assegurar a privacidade do tratamento & $2(2,40 \%)$ & $1(1,20 \%)$ & $82(96,40 \%)$ & 4,36 \\
\hline 6. Confidencialidade das informações & $2(2,40 \%)$ & $3(3,50 \%)$ & $80(94,10 \%)$ & 4,43 \\
\hline 7. Competência do profissional & - & $5(5,90 \%)$ & $80(94,10 \%)$ & 4,64 \\
\hline 8. Beneficiou-se com o tratamento & $1(1,20 \%)$ & $6(7,10 \%)$ & $78(91,70 \%)$ & 5,29 \\
\hline
\end{tabular}


baixas frequências de resposta. Os vários tipos de diagnósticos dos pacientes, que estavam sob cuidado desses familiares, foram agrupados em três categorias, de acordo com a CID-1027: (1) "Esquizofrenia, Transtornos esquizotípicos e Transtornos delirantes", (2) "Transtornos do humor e afetivos", e (3) "Transtornos neuróticos relacionados ao estresse e somatoforme". A categoria diagnóstica Transtornos fóbicos ansiosos não foi analisada, porque tinha apenas um sujeito. Para a variável "local de tratamento", foram comparados os dados de apenas dois serviços, ambulatório e CAPS, pois so- mente quatro pacientes faziam tratamento em um terceiro serviço.

A tabela 5 apresenta os resultados referentes à análise das variáveis categóricas sociodemográficas e clínicas, associadas à satisfação dos familiares. Nessa tabela, para comparar duas categorias, foi utilizado o teste Man-Whitney e, para comparar mais de duas categorias, foi utilizado o teste de Kruskal-Wallis. A tabela 6 apresenta a análise das variáveis contínuas sociodemográficas e clínicas associadas à satisfação dos familiares, por meio do coeficiente de correlação de Spearman.

Tabela 5. Análise univariada das variáveis categóricas sociodemográficas e clínicas associadas à satisfação global dos familiares com os serviços, por meio dos testes Man-Whitney e Kruskal-Wallis

\begin{tabular}{|c|c|c|c|c|}
\hline Variáveis & Categorias & N & Média (DP) & $P$ \\
\hline Gênero do paciente & $\begin{array}{l}\text { Feminino } \\
\text { Masculino }\end{array}$ & $\begin{array}{l}68 \\
17\end{array}$ & $\begin{array}{l}4,46(0,63) \\
4,34(0,50)\end{array}$ & 0,58 \\
\hline Paciente frequentou escola & $\begin{array}{l}\text { Sim } \\
\text { Não }\end{array}$ & $\begin{array}{c}80 \\
5\end{array}$ & $\begin{array}{l}4,38(0,57) \\
4,80(0,19)\end{array}$ & 0,12 \\
\hline Renda própria (paciente) & $\begin{array}{l}\text { Sim } \\
\text { Não }\end{array}$ & $\begin{array}{l}53 \\
32\end{array}$ & $\begin{array}{l}4,46(0,55) \\
4,34(0,57)\end{array}$ & 0,24 \\
\hline \multirow{3}{*}{ Categoria diagnóstica } & $\begin{array}{l}\text { - Esquizofrenia, Transtornos Esquizotípicos e Transtornos Delirantes } \\
\text { - Transtornos do Humor e Afetivos }\end{array}$ & $\begin{array}{l}51 \\
23\end{array}$ & $\begin{array}{l}4,40(0,60) \\
4,44(0,54)\end{array}$ & 0,93 \\
\hline & $\begin{array}{l}\text { Esquizofrenia, transtornos esquizotípicos e transtornos delirantes } \\
\text { Transtornos neuróticos relacionados ao estresse e somatoforme }\end{array}$ & $\begin{array}{c}51 \\
8\end{array}$ & $\begin{array}{l}4,40(0,60) \\
4,50(0,42)\end{array}$ & 0,99 \\
\hline & $\begin{array}{l}\text { Transtornos do humor e afetivo } \\
\text { Transtornos neuróticos relacionados ao estresse e somatoforme }\end{array}$ & $\begin{array}{c}23 \\
8\end{array}$ & $\begin{array}{l}4,44(0,54) \\
4,50(0,42)\end{array}$ & 0,96 \\
\hline Comorbidade psiquiátrica & $\begin{array}{l}\text { Sim } \\
\text { Não }\end{array}$ & $\begin{array}{l}15 \\
69\end{array}$ & $\begin{array}{l}4,28(0,58) \\
4,44(0,56)\end{array}$ & 0,20 \\
\hline Local de tratamento & $\begin{array}{l}\text { Ambulatório } \\
\text { CAPS }\end{array}$ & $\begin{array}{l}46 \\
35\end{array}$ & $\begin{array}{r}4,41(0,51) \\
4,44(0,60)\end{array}$ & 0,41 \\
\hline Modalidade de tratamento & $\begin{array}{l}\text { CAPS DIA } \\
\text { Ambulatorial }\end{array}$ & $\begin{array}{l}37 \\
48\end{array}$ & $\begin{array}{l}4,37(0,51) \\
4,44(0,59)\end{array}$ & 0,31 \\
\hline Paciente possui doenças físicas & $\begin{array}{l}\text { Sim } \\
\text { Não }\end{array}$ & $\begin{array}{l}38 \\
47\end{array}$ & $\begin{array}{l}4,33(0,56) \\
4,48(0,55)\end{array}$ & 0,14 \\
\hline Paciente faz outro tratamento de saúde & $\begin{array}{l}\text { Sim } \\
\text { Não }\end{array}$ & $\begin{array}{l}35 \\
50\end{array}$ & $\begin{array}{l}4,41(0,51) \\
4,41(0,59)\end{array}$ & 0,76 \\
\hline Paciente internou-se & $\begin{array}{l}\text { Sim } \\
\text { Não }\end{array}$ & $\begin{array}{l}54 \\
31\end{array}$ & $\begin{array}{l}4,43(0,57) \\
4,38(0,54)\end{array}$ & 0,48 \\
\hline Paciente toma o medicamento sozinho & $\begin{array}{l}\text { Sim } \\
\text { Não }\end{array}$ & $\begin{array}{l}56 \\
29\end{array}$ & $\begin{array}{l}4,37(0,60) \\
4,49(0,47)\end{array}$ & 0,55 \\
\hline Tipo de medicação & $\begin{array}{l}\text { Via oral } \\
\text { Oral e intramuscular }\end{array}$ & $\begin{array}{l}68 \\
17\end{array}$ & $\begin{array}{l}4,46(0,52) \\
4,21(0,67)\end{array}$ & 0,17 \\
\hline Gênero do familiar & $\begin{array}{l}\text { Feminino } \\
\text { Masculino }\end{array}$ & $\begin{array}{l}68 \\
17\end{array}$ & $\begin{array}{l}4,40(0,60) \\
4,47(0,38)\end{array}$ & 0,81 \\
\hline Familiar frequentou escola & $\begin{array}{l}\text { Sim } \\
\text { Não }\end{array}$ & $\begin{array}{l}74 \\
11\end{array}$ & $\begin{array}{l}4,43(0,58) \\
4,30(0,36)\end{array}$ & 0,17 \\
\hline Renda própria do familiar & $\begin{array}{l}\text { Sim } \\
\text { Não }\end{array}$ & $\begin{array}{l}53 \\
32\end{array}$ & $\begin{array}{l}4,42(0,55) \\
4,41(0,58)\end{array}$ & 0,83 \\
\hline Trabalho formal (familiar) & $\begin{array}{l}\text { Sim } \\
\text { Não }\end{array}$ & $\begin{array}{l}53 \\
32\end{array}$ & $\begin{array}{l}4,58(0,48) \\
4,39(0,57)\end{array}$ & 0,32 \\
\hline Familiar reside com o paciente & $\begin{array}{l}\text { Sim } \\
\text { Não }\end{array}$ & $\begin{array}{l}12 \\
73\end{array}$ & $\begin{array}{l}4,39(0,54) \\
4,57(0,63)\end{array}$ & 0,11 \\
\hline Estado civil do familiar & $\begin{array}{l}\text { Solteiro } \\
\text { Casado } \\
\text { Vivendo como casado } \\
\text { Separado } \\
\text { Viúvo }\end{array}$ & $\begin{array}{c}11 \\
49 \\
2 \\
7 \\
16\end{array}$ & $\begin{array}{l}4,42(0,52) \\
4,47(0,52) \\
4,56(0,27) \\
4,11(0,53) \\
4,34(0,72)\end{array}$ & 0,60 \\
\hline Parentesco do familiar com o paciente & $\begin{array}{l}\text { Mãe/pai } \\
\text { Irmão } \\
\text { Cônjuge } \\
\text { Filho } \\
\text { Outro }\end{array}$ & $\begin{array}{l}37 \\
17 \\
15 \\
8 \\
8\end{array}$ & $\begin{array}{l}4,33(0,60) \\
4,62(0,46) \\
4,31(0,48) \\
4,47(0,54) \\
4,52(0,70)\end{array}$ & 0,20 \\
\hline
\end{tabular}


Os resultados das tabelas 5 e 6 mostraram que, de todas as variáveis analisadas, somente a idade do paciente e o tempo transcorrido desde a sua última internação estavam significativamente associados ao grau de satisfação dos familiares. Os familiares apresentaram maior grau de satisfação com os serviços quando os pacientes eram mais velhos ( $r=$ 0,$23 ; p=0,04$ ) e quando estavam há mais tempo, medido em meses, sem passar por uma internação psiquiátrica $(r=0,26$; $p=0,05)$. Nenhuma variável sociodemográfica dos familiares estava associada à sua satisfação com os serviços de saúde mental avaliados

Tabela 6. Análise univariada das variáveis contínuas sociodemográficas e clínicas associadas à satisfação global dos familiares com os serviços, por meio da correlação de Spearman

\begin{tabular}{lccc}
\hline Variáveis & Média (DP) & $\mathbf{R}$ & $\mathbf{P}$ \\
\hline Idade do paciente & $43,53(12,49)$ & 0,23 & $0,04^{*}$ \\
Número de anos de escolaridade do paciente & $4,99(3,34)$ & $-0,18$ & 0,10 \\
Idade do início do transtorno psiquiátrico & $27,10(11,88)$ & 0,04 & 0,74 \\
Duração do transtorno & $16,89(10,32)$ & 0,20 & 0,08 \\
Duração do tratamento psiquiátrico & $13,02(9,33)$ & 0,18 & 0,09 \\
Número médio de internações & $7,42(18,00)$ & 0,06 & 0,65 \\
Duração da última internação & $55,91(67,66)$ & 0,06 & 0,64 \\
Tempo desde a última internação (em meses) & $96,85(93,60)$ & 0,26 & $0,05 *$ \\
Número de crises no último ano & $2,74(2,38)$ & 0,03 & 0,82 \\
Número de tipos de medicamentos & $2,99(1,21)$ & $-0,05$ & 0,62 \\
Idade do familiar & $54,61(14,38)$ & $-0,01$ & 0,94 \\
Número de anos de escolaridade do familiar & $4,98(3,34)$ & 0,00 & 0,99 \\
Renda do familiar (em reais) & $555,06(254,18)$ & $-0,09$ & 0,53 \\
\hline * p $\leq 0,05$. & & &
\end{tabular}

${ }^{*} p \leq 0,05$.

\section{DISCUSSÃO}

Os resultados do presente estudo mostraram que a grande maioria dos familiares (entre 81,20\% e 96,40\%) demonstrou satisfação ou muita satisfação em relação aos diferentes aspectos dos serviços. Uma minoria (1,20\% a 5,90\%) relatou estar insatisfeita ou muito insatisfeita, e uma porcentagem um pouco maior (1,20\% a 16,50\%) apresentou a resposta "mais ou menos", intermediária entre satisfação e insatisfação.

Dois estudos internacionais encontraram taxas mais baixas de satisfação dos familiares com os serviços de saúde mental, comparativamente ao presente estudo. Embora essa diferença de resultados possa estar relacionada com o tipo de serviço, sua organização e tratamentos envolvidos, não é possível fazer uma comparação nesse nível, uma vez que os autores não apresentaram descrições detalhadas sobre esses aspectos. Outras hipóteses, entretanto, podem ser levantadas para explicar as diferenças entre os resultados dos estudos. Na pesquisa de Perreault et al. ${ }^{7}$, as porcentagens mais baixas de satisfação ou muita satisfação encontradas $(46,40 \%$ a $85,70 \%)$ talvez se expliquem pelo tipo de amostra avaliada, pois se tratava de familiares que faziam parte de grupos de suporte, portanto possivelmente mais organizados, informados e engajados em movimentos coletivos do que os familiares da amostra brasileira avaliada, o que poderia talvez contribuir para uma manifestação mais aberta de opiniões, expectativas e críticas. Além disso, a forma de coletar os dados, por meio de entrevistas por telefone, talvez tenha contribuído também para uma maior manifestação de insatisfação do que entrevistas face a face, tal como utilizada no presente trabalho.

No estudo de Stengard et al. ${ }^{8}$, os familiares foram entrevistados em uma avaliação de follow-up após a alta de internação, sendo que dois terços da amostra demonstraram satisfação com os serviços, enquanto um terço apresentou insatisfação, particularmente com a falta de sua participação no tratamento e com a falta de informações por parte dos profissionais dos serviços. Neste estudo, o tipo de amostra também pode ter contribuído para uma maior manifestação de insatisfação, pois se tratava de 60\% dos familiares da amostra total do estudo, cujos pacientes estavam em condições clínicas mais desfavoráveis do que o restante da amostra total, uma vez que usavam mais medicamentos, frequentavam mais os serviços e apresentavam menor grau de autonomia. Além disso, tratava-se de pacientes que tinham recebido alta dos hospitais há apenas três anos, possivelmente ainda em processo de readaptação. No presente estudo, ao contrário, a amostra era constituída por pacientes estabilizados, que estavam em regime ambulatorial, viviam na comunidade e tinham uma média de 17,89 anos de duração da doença, e a maioria (51,80\%) tinha tido apenas uma ou nenhuma internação, há 96,85 meses em média. No último ano, a maioria (75,30\%) tinha sofrido apenas uma ou nenhuma crise. A presente amostra apresenta, portanto, características diferentes das amostras dos dois estudos citados acima, que talvez expliquem as diferenças de resultados da satisfação dos familiares.

No que se refere às condições clínicas dos pacientes, já foi constatado que essa variável está associada à satisfação dos familiares com os serviços. Por exemplo, Stengard et al. ${ }^{8}$ encontraram que familiares de pacientes com melhor funcionamento global estavam mais satisfeitos com os serviços de saúde mental $(O R=0,73 ; p=0,003)$. O estudo de Perreault et al. ${ }^{7}$ também mostrou que a satisfação dos familiares estava significativamente associada ao maior tempo de duração da doença do paciente ( $r=0.21$ e $p=0.014)$, o que sugere estabilidade do quadro clínico dos pacientes. No presente estudo, a maior satisfação dos familiares estava correlacionada com duas variáveis dos pacientes: maior tempo transcorrido desde a última internação e maior idade, o que também sugere uma estabilidade do quadro clínico. Na presente amostra, os pacientes com maior idade eram também aqueles 
que possuíam o transtorno psiquiátrico há mais tempo $(r=$ 0,39, $p<0,001$ ), que faziam tratamento psiquiátrico por um período mais longo ( $r=0,37, p \leq 0,001)$ e que estavam há mais tempo sem internação $(r=0,54, p<0,001)$.

Outra hipótese explicativa dos resultados da presente pesquisa pode ser levantada examinando a realidade dos serviços de saúde do país, comparativamente aos serviços de saúde mental e os efeitos dessa comparação nas expectativas e apreciações dos familiares dos pacientes psiquiátricos. A alta satisfação dos familiares do presente trabalho talvez se explique à luz do modelo da expectativa, em particular a teoria do contraste, descrito na introdução deste artigo. Segundo esse modelo, os sujeitos avaliam um determinado serviço, com base em uma comparação que fazem entre a experiência atual com o serviço e experiências prévias com outros serviços de saúde. No presente estudo, uma comparação entre as experiências prévias dos familiares com os serviços de saúde em geral, tais como os postos de saúde, nos quais eles enfrentam filas de espera longas para conseguir uma consulta, em contraste com a de um serviço de saúde mental, como os CAPS, apontaria para as vantagens desse último serviço. No estudo de Silva ${ }^{28}$, os próprios pacientes haviam relatado que, nos CAPS, eles não necessitam enfrentar filas de espera para marcar consultas, além de terem medicamentos gratuitos, e que eles se sentiam satisfeitos com os serviços de saúde mental por causa dessas suas características. Essa interpretação é coerente com os resultados de outros estudos brasileiros com pacientes psiquiátricos, que também relataram alto nível de satisfação desses pacientes com os serviços ${ }^{17-19}$.

No entanto, outro resultado da presente pesquisa, que merece comentários, foi o fato de a variável serviço não constituir um fator associado ao grau de satisfação dos familiares, embora se tratasse de dois tipos teoricamente diferentes de serviço, CAPS e ambulatório. Esses resultados são surpreendentes, pois serviços definidos por objetivos e organizações distintas tenderiam a proporcionar níveis diferenciados de satisfação dos familiares, por exemplo, quando esses serviços desenvolvem intervenções diferentes ${ }^{7}$. Os CAPS deveriam, em princípio, ser diferentes dos ambulatórios, em termos da diversidade de intervenções e da atenção mais frequente e intensiva aos pacientes e seus familiares, podendo gerar níveis diferentes de satisfação. Os resultados encontrados no presente trabalho talvez se expliquem pela semelhança de tratamento oferecido aos pacientes da presente amostra, independentemente do tipo de serviço que frequentavam. Como se tratava de pacientes estabilizados, o tratamento se limitou ao medicamento e consultas médicas infrequentes, em ambos os tipos de serviço. Esses dados sugerem uma realidade peculiar dos serviços de saúde mental avaliados e apontam para a necessidade de maior diferenciação entre eles. Destaca-se, em particular, a necessidade de maior desenvolvimento, nos CAPS, das ações previstas de intervenções psicoeducacionais de grupo, com os familiares e pacientes e visitas domiciliares. Uma tendência à "ambulatorização" dos CAPS já foi apontada em outras localidades, assim como a escassez de intervenções voltadas para a reinserção social dos pacientes, o que compromete a qualidade do atendimento em saúde mental ${ }^{29}$.

O presente estudo apresenta a limitação de ter incluído uma amostra pequena, e do tipo não aleatório, de familiares participantes. Amostras não aleatórias podem influenciar os resultados em favor de um maior grau de satisfação dos familiares com os serviços. O tamanho reduzido da amostra pode também ter influenciado o resultado referente à falta de diferenciação entre os serviços. Pesquisas futuras com amostras aleatórias e de maior tamanho poderão reavaliar os resultados obtidos.

\section{CONCLUSÃO}

Os dados destacaram a elevada porcentagem de satisfação dos familiares com os serviços de saúde mental, o que talvez se explique pelo contraste cognitivo com outros serviços de saúde. Dentre os fatores associados à satisfação, destacou-se a influência das variáveis dos pacientes, em particular suas condições clínicas, no grau de satisfação dos familiares cuidadores. O nível de satisfação não diferiu entre dois tipos teoricamente diversos de serviço, CAPS e ambulatório, provavelmente devido a semelhanças do tratamento oferecido nesses serviços. Esses resultados apontam para a necessidade de maior diferenciação entre os serviços, porém tais dados devem ser reavaliados em estudos futuros, com amostras maiores.

\section{CONFLITO DE INTERESSES}

Nenhum.

\section{FINANCIAMENTO}

Fundação de Amparo à Pesquisa do Estado de Minas Gerais (Fapemig) e Coordenação de Aperfeiçoamento de Pessoal de Nível Superior (Capes).

\section{REFERÊNCIAS}

1. World Health Organization. The World Health Report, Mental Health: New Understanding, New Hope; 2001

2. Hamson L. Outcome assessment in psychiatric service evaluation. Soc Psychiatry Psychiatric Epidemiol. 2001;36:244-8.

3. Donabedian A. Quality assurance in health care: consumers' role. Qual Health Care. 1992;:1:247-51.

4. Mercier L, Landry M, Corbière M, Perreault M. Measuring clients perception as outcome measurement. In: Roberts AR, Yeager KR, orgs. Evidence-based Practice Manual: Research 
an outcome Measures in Health and Human Services. New York: Oxford University Press; 2004. p. 904-9.

5. Hasler G, Moergeli H, Schnyder U. Outcomes of psychiatric treatment: what is relevant for our patients? Compr Psychiatry. 2004;45(3):199-205.

6. Hanson JG, Rapp CA. Families' perceptions of community mental health programs for their relatives with a severe mental illness. Community Ment Health J. 1992;28(3):181-97.

7. Perreault M, Rousseau M, Provencher H, Roberts S, Milton D. Predictors of caregivers' satisfaction in mental health services. Community Ment Health J. 2011:1-6.

8. Stengard EL, Honkonen T, Koivisto AM, Salokangas RQR. Satisfaction of caregivers of patients with schizophrenia in Finland. Psychiatr Serv. 2000;51:1034-9.

9. Tessler RC, Gamache GM. Family experiences with mental illness. Westport: Auburn House; 2000.

10. Bandeira M, Barroso SM. Sobrecarga das famílias de pacientes psiquiátricos. J Bras Psiquiatr. 2005;54(1):34-46

11. Ruggeri M. Patients' and relatives' satisfaction with psychiatric services: the state of the art of its measurement. Soc Psychiatr Epidem. 1994;29:212-27.

12. Hasler G, Moergeli H, Bachmann R, Lambreva E, Buddeberg C, Schnyder U. Patient satisfactions with outpatient psychiatric treatment: the role of diagnosis, pharmacotherapy, and perceived therapeutic change. Can J Psychiatry. 2004;49(5):315-21.

13. Pribe S, Gruyters T. Patients' assessment of treatment predicting outcome. Schizophr Bull. 1995;21:87-94.

14. Pascoe GC. Patient satisfaction in primary health care: a literature review and analysis. Eval Program Plann. 1993;6:185-210

15. Gigantesco A, Picardi A, Chiaia E, Balbi A, Morosine P. Patients' satisfaction with psychiatric services in a large catchment area in Rome. Eur Psychiatry. 2002;17:139-47.

16. Bandeira M, Mercier C, Perreault M, Libério MMA, Pitta AMF. Escala de avaliação da satisfação dos familiares com os serviços de saúde mental: Satis-BR. J Bras Psiquiatr. 2002:51(3):153-66

17. Jaegger RC, Guitton AP, Lyrio JM, Santos MM, Freitas RCO, Gonçalves SR, et al. A experiência de morar fora: avaliação da satisfação de usuários em um serviço de saúde mental. Cad Saúde Col. 2004;12(1):27-39.
18. Heckert U, Teixeira LS, Trindade AS. Avaliação da satisfação dos usuários do Centro Regional de Referência em Saúde Mental (CRRESAM) da região central de Juiz de Fora, MG. HU Rev. 2006;32(1):15-9.

19. Kantorski LP, Jardim VR, Wetzel C, Olschowsky A, Schneider JF, Heck RM. Satisfação dos usuários dos centros de atenção psicossocial da região Sul do Brasil. Rev Saude Publica. 2009;43(1):29-35.

20. Selltiz C, Wrightsman LS, Cook SW. Métodos de pesquisa nas relações sociais. São Paulo: EPU; 1987.

21. Contandriopoulos AP, Champagne F, Potvin L, Denis JL, Bouyle P. Saber preparar uma pesquisa. São Paulo: Hucitec Abrasco; 1994.

22. World Health Organization. WHO-SATIS consumer's and caregivers' satisfaction with mental health services a multisite study. Geneva: Mental Health World Organization; 1996.

23. Vallerand RJ. Vers une méthodologie de validation trans-culturelle de questionnaires psychologiques: implications pour la recherché en langue française. Can Psychol. 1989;30(4):662-80

24. Guillemin F, Bombardier C, Beaton D. Cross-cultural adaptation of health-related quality of life measures: literature review and proposed guidelines. J Clin Epidem. 1993;46(12):1417-32.

25. Bisqueira R, Sarriera JC, Martínez F. Introdução à estatística: enfoque informático com 0 pacote estatístico SPSS. Porto Alegre: Artmed; 2004.

26. Dancey (P, Reidy J. Estatística sem matemática para Psicologia. Usando SPSS para Windows. Porto Alegre: Artmed; 2006

27. Organização Mundial de Saúde. (ID-10: classificação internacional de doenças e problemas relacionados à saúde. São Paulo: Edusp; 1998.

28. Silva MA. Percepção de mudança em função do tratamento e satisfação dos pacientes psiquiátricos em serviços de saúde mental [dissertação]. São João del-Rei (MG): Universidade Federal de São João del-Rei; 2011

29. Nilo K, Moraes MAB, Guimarães MBL, Vasconcelos ME, Nogueira MTG, Abou-Yd M. Política de saúde mental de Belo Horizonte: o cotidiano de uma utopia. Belo Horizonte (MG): Secretaria Municipal de Saúde de Belo Horizonte; 2008. 258p. 